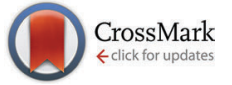

Cite this: J. Mater. Chem. C, 2016, 4, 5634

\title{
Inkjet printing of uniform dielectric oxide structures from sol-gel inks by adjusting the solvent composition
}

\author{
A. Matavž, ${ }^{\star a b}$ R. C. Frunză, ${ }^{a}$ A. Drnovšek, ${ }^{a b}$ V. Bobnar ${ }^{a b}$ and B. Malič ${ }^{a b}$
}

\begin{abstract}
We present a study of the inkjet printing of tantalum-oxide-based dielectric structures on indium-tinoxide-coated glass. Ta-Al-Si-alkoxide-based ink formulations with 2-methoxyethanol (2MOE) as the main solvent and a highly viscous glycerol (GLY) or 1,3-propanediol (PD) co-solvent exhibit the optimal values of viscosity and surface tension for piezoelectric inkjet printing, and show good jetting performance. However, the drying of the printed structures results in a pronounced "coffee-stain" effect. We relate this phenomenon to the much higher volatility of $2 \mathrm{MOE}$ than either of the viscous solvents and the dominant evaporation of the former immediately after the ink has been printed on the substrate. Consequently, the recirculating Marangoni solvent flow exists only at the onset of drying and ceases to exist once the $2 \mathrm{MOE}$ has completely evaporated from the drying feature. The combination of all three solvents appears to prolong the duration of the Marangoni flow, as suggested by differential scanning calorimetry, resulting in an improved uniformity of dried structures. By adjusting the solvent composition we could tailor the topology of deposits and print $45 \mathrm{~nm}$-thick, flat and uniform capacitors with the performance (good dielectric properties of $\varepsilon_{\mathrm{r}} \sim 15, \tan (\delta) \sim 0.034$ at $100 \mathrm{kHz}$ and a low leakage current density of $2.4 \times 10^{-7} \mathrm{~A} \mathrm{~cm}^{-2}$ at $200 \mathrm{kV} \mathrm{cm}^{-1}$ ) comparable to spin-coated films.
\end{abstract}

Received 14th March 2016, Accepted 13th May 2016

DOI: $10.1039 / c 6 t c 01090 c$

www.rsc.org/MaterialsC

\section{Introduction}

The increasing demand to produce low-cost, large-area, flexible and lightweight electronic devices has promoted the rapid development of printed electronics. ${ }^{1}$ Inkjet printing offers the possibility of automation, and a resolution in the micrometre range. ${ }^{2,3}$ In the inkjet printing process, the picolitre-sized droplets of ink are ejected from the print-head nozzle and deposited onto a substrate at digitally determined locations. The process is computer-guided, highly flexible, and enables direct patterning of functional materials without the use of any additional patterning steps, such as lithography. ${ }^{4,5}$

The physical properties of the ink that enable stable drop formation in piezoelectric inkjet printing, i.e. viscosity, surface tension, and density, are well defined and related through the dimensionless Ohnesorge number. For proper drop formation, the inverse of the Ohnesorge number, or $Z$ number, (eqn (1)) of the ink should be in the range of $1-14 .{ }^{6,7}$ It is defined as:

$$
Z=\frac{\operatorname{Re}}{\sqrt{\mathrm{We}}}=\frac{\sqrt{a \rho \gamma}}{\eta}
$$

\footnotetext{
${ }^{a}$ Jožef Stefan Institute, Jamova cesta 39, 1000 Ljubljana, Slovenia.

E-mail: aleksander.matavz@ijs.si; Tel: +38614773832

${ }^{b}$ Jožef Stefan International Postgraduate School, Jamova cesta 39, 1000 Ljubljana, Slovenia
}

where Re is the Reynolds number, We is the Weber number, $a$ is the nozzle diameter, $\rho$ is the density, $\gamma$ is the surface tension, and $\eta$ is the viscosity of the fluid.

After the ink has been printed onto a non-porous substrate, it is dried and annealed to obtain a thin solid film. The ink often dries so that a ring-like deposit forms, a phenomenon often referred to as the "coffee-stain" effect. This effect is commonly observed for drops with a pinned contact line, for which the evaporation rate is the highest at the edge and the pinning prevents the contraction of the drop. The flow of the liquid from the centre towards the edges of the drop results in a build-up of the edge deposit. ${ }^{8}$ The morphology strongly impacts the performance of the printed structures, ${ }^{9,10}$ and thus the ability to precisely control the morphology is essential for the fabrication of novel, high-performance devices. ${ }^{11}$ Recent reports show that several factors can influence the morphology of a dried deposit, including the drying temperature, ${ }^{12,13}$ the ambient humidity, ${ }^{9}$ the presence of the solvent vapour, ${ }^{14}$ and the shape of the particles in a suspension. ${ }^{15}$

It has also been shown that a recirculating Marangoni flow can improve the uniformity of deposits. ${ }^{16,17}$ The flow appears in the presence of a surface-tension gradient; it can be induced by surfactants, thermal fluctuations and/or a concentration gradient. An elegant approach to induce the Marangoni flow in printed structures is based on rigorously selecting the solvent 
composition of the ink. The flow is generated as the more volatile solvent evaporates faster from the edge of the drying feature, resulting in a concentration gradient, and consequently a surface-tension gradient if the solvents have different surface tensions. The effectiveness of the solvent-induced Marangoni flow significantly varies for particular ink formulations, and appears to be mostly dependent on the choice of the solvents, their volume fractions, and the concentration of a solute. ${ }^{18}$ In order to ensure a stable drop formation, diluted inks suitable for the deposition of ultra-thin patterns commonly contain a large fraction of viscous solvents with high-boiling points. Drying of such inks can result in the "coffee-stain" effect as the Marangoni flow becomes suppressed if a more volatile solvent evaporates completely from the drying deposit long-before the drying is finished. Therefore, understanding how the Marangoni flow evolves during the drying process and how it influences the topology of the deposit is required.

In this study we focused on the printing of high-dielectricconstant metal-oxide layers, which are among the essential building blocks for electronic components such as thin-film capacitors and field-effect transistors for transparent electronics. The dielectric tantalum(v) oxide has a high dielectric constant ( $\sim 25$ in the amorphous phase), a high refractive index, and a high chemical stability. ${ }^{19}$ Its relatively large leakage current is effectively reduced by incorporating metal oxides with a larger band gap, e.g. $\mathrm{Al}_{2} \mathrm{O}_{3}$ and/or $\mathrm{SiO}_{2} \cdot{ }^{20,21}$ The homogeneity of the electric field across the dielectric layer is crucial for a proper operation, and any variations in the thickness and thickness uniformity would result in a deterioration of the performance.

We show that besides the design of the solvent mixture based on the viscosity and surface-tension criteria, the volatility of the solvents in the ink strongly influences the thickness uniformity of the deposit, and consequently the performance of the printed capacitors.

\section{Experimental}

\section{Synthesis of the inks and the printing process}

Tantalum ethoxide (H. C. Starck, 99.99\%), aluminum-tri-secbutoxide (Sigma Aldrich, 97\%) and tetraethoxysilane (Alfa Aesar, 99.9\%) were used as $\mathrm{Ta}_{2} \mathrm{O}_{5}, \mathrm{Al}_{2} \mathrm{O}_{3}$ and $\mathrm{SiO}_{2}$ precursors, respectively. The precursor solutions, all with a $0.1 \mathrm{M}$ concentration, were prepared separately at room temperature in an inert nitrogen atmosphere, as previously described, ${ }^{20}$ and mixed in an $8: 1: 1$ molar ratio (subsequently referred to as the TAS solution). The volume fractions of the solvents 2-methoxyethanol (Alfa Aesar, 99.3+\%) and ethanol (Merck, 99.9+\%) in the TAS solution were $90 \mathrm{vol} \%$ and $10 \mathrm{vol} \%$, respectively. The inks were prepared by admixing 1,3-propanediol (Sigma Aldrich, 98\%) and/or glycerol (Alfa Aesar, 99.95\%) to the TAS precursor solution. The final concentration of the inks was $0.05 \mathrm{M}$ (corresponding to the $1.85 \mathrm{wt} \%$ of load), except for the ink containing $55 \mathrm{vol} \%$ of 1,3-propanediol, for which the final concentration was $0.045 \mathrm{M}$ (corresponding to $1.67 \mathrm{wt} \%$ of load).
The inkjet printing was performed under ambient conditions using a Dimatix DMP 2831 piezoelectric printer equipped with a $10 \mathrm{pL}$ cartridge (DMC-11610). The printing parameters were as follows: a signal voltage of $21 \mathrm{~V}$, a jetting frequency of $20 \mathrm{kHz}$, a distance between the cartridge and the substrate of $0.7 \mathrm{~mm}$, and a drop spacing of $20 \mu \mathrm{m}$. During the printing, the temperature of the printer platen and the nozzles was set to ambient $\left(22-23{ }^{\circ} \mathrm{C}\right)$. The printing pattern consisted of an array of $15 \times 15$ squares, each with dimensions of $500 \times 500 \mu^{2}$, with a $250 \mu \mathrm{m}$ separation between the squares. The patterns were printed onto indium-tin-oxide (ITO) covered glass substrates $\left(R_{\mathrm{S}} \leq 200 \Omega \square^{-1}\right)$ and dried immediately after the printing process by heating the samples in air on a hot plate at $150{ }^{\circ} \mathrm{C}$ for $10 \mathrm{~min}$. After the printing and drying step, the deposited organic precursors were pyrolyzed by heating the samples at $350{ }^{\circ} \mathrm{C}$ for $10 \mathrm{~min}$.

\section{Characterization of the inks and printed patterns}

The physical properties of the inks were measured at $25{ }^{\circ} \mathrm{C}$ using a Physica MCR 301 rheometer and a Krüss EasyDrop tensiometer was used to record the drops during drying at $150{ }^{\circ} \mathrm{C}$ from the side-view. Photos of the sessile drops were recorded at different times and analysed using Krüss software. The evaporation process of the inks was investigated using a Netzsch DSC 204 F1 calorimeter in an air atmosphere with a flow of $20 \mathrm{~mL} \mathrm{~min} \mathrm{~m}^{-1}$ and a heating rate of $10 \mathrm{~K} \mathrm{~min}^{-1}$. We used pierced aluminum crucibles with a hole diameter of $\sim 150 \mu \mathrm{m}$. The typical sample mass was $\sim 10 \mathrm{mg}$.

The surface morphology of the printed patterns was investigated using a Bruker Dektak XT profilometer and an Asylum Research MFP-3D-STM atomic force microscope. Thin-film capacitors in the metal-insulator-metal (MIM) configuration were fabricated by sputtering ( 5 Pascal) circular gold electrodes of $200 \mu \mathrm{m}$ diameter using a shadow mask, followed by heating at $150{ }^{\circ} \mathrm{C}$ for $10 \mathrm{~min}$. The dielectric response of the capacitors was measured in the frequency range of $100 \mathrm{~Hz}$ to $1 \mathrm{MHz}$ using an HP 4284A impedance analyser and a signal amplitude of $50 \mathrm{mV}$. The current-voltage characteristics were measured using a Keithley 237 with a voltage step of $100 \mathrm{mV}$, a $3 \mathrm{~s}$ hold time and a $2 \mathrm{~s}$ measurement time for each voltage step.

\section{Results and discussion}

\section{Solvent mixtures}

The first step in inkjet printing is the preparation of an ink with suitable physical properties. While 2-methoxyethanol (2MOE) is an excellent solvent for metal alkoxides, its viscosity (1.6 mPa s) is much too low for stable drop formation during inkjet printing. We increased the viscosity of the $2 \mathrm{MOE}$ by admixing a more viscous solvent, 1,3-propanediol (PD, $\eta \approx 42 \mathrm{mPa} \mathrm{s}$ ) or glycerol (GLY, $\eta \approx 830 \mathrm{mPa} \mathrm{s}$ ). ${ }^{22,23}$ The physical properties of the respective solvents are collected in Table 1.

The viscosity behaviour of the 2MOE-GLY and 2MOE-PD solvent mixtures is presented in Fig. 1a. Due to the higher viscosity of the GLY, compared to the PD (see Table 1), the 
Table 1 Properties of 2-methoxyethanol, 1,3-propanediol and glycerol

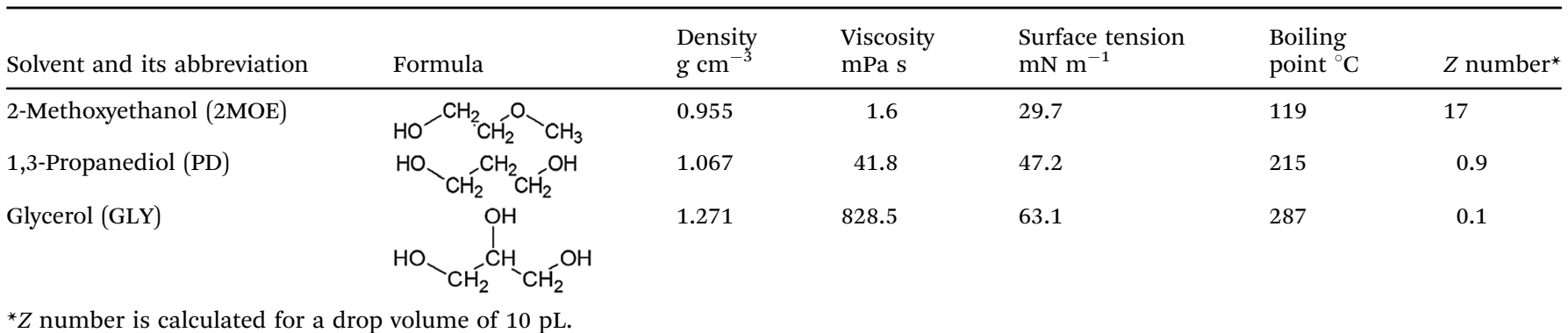

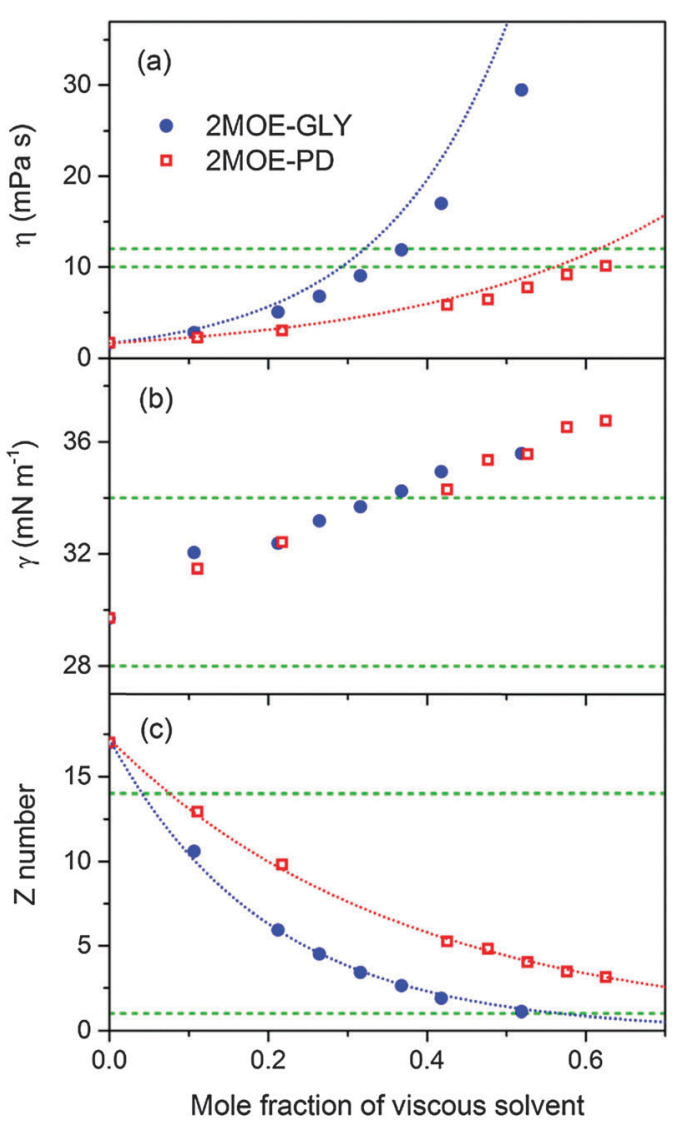

Fig. 1 (a) Viscosity, (b) surface tension and (c) $Z$ number of the solvent mixtures as a function of the added viscous component. The dotted curves in (a) correspond to the values calculated using eqn (2). The optimum ranges of both parameters, as recommended by the printer producer, ${ }^{24}$ are given within the horizontal dashed lines.

viscosity of the respective mixtures increases faster than the viscosity of the 2MOE-PD mixtures and reaches optimal values at lower fractions of the admixed viscous solvent, i.e. about $10 \mathrm{mPa}$ s for fractions of 30 vol\% GLY and 55 vol\% PD. In an ideal system, the exponential increase of the viscosity with the fraction of the viscous solvent is predicted as:

$$
\ln \eta=x_{1} \ln \left(\eta_{1}\right)+x_{2} \ln \left(\eta_{2}\right)
$$

where $\eta$ is the viscosity, $x$ is the molar fraction, and subscripts 1 and 2 denote the first and the second solvent, respectively. ${ }^{25}$ If we compare the viscosity of our solvent mixtures with the viscosity of such an ideal system, we observe that both solvent mixtures show a small negative deviation from the theoretical values.

The surface tension of both solvent mixtures increases slightly with an increasing fraction of the viscous solvent, see Fig. 1b. The simultaneous strong increase of viscosity and only a slight increase of surface tension are reflected in a decrease of the $Z$ number, which according to eqn (1) is proportional to $\sqrt{\gamma} / \eta$, Fig. 1c. The viscosity and surface-tension measurements suggest that adding 30 or 55 vol\% of the GLY or PD to the $2 \mathrm{MOE}$, respectively, should match the printing requirements.

\section{Formulation of the sol-gel inks: printing and drying behaviour}

Thus far we have discussed the physical properties of the solvent mixtures without any solid load. Upon dissolving Ta-, Al-, and Si-alkoxides in the solvent mixtures (the solutions are subsequently referred to as inks), the viscosity slightly increased as compared to the respective solvent mixtures (cf. Fig. 1a and Table 2), while the surface tension and the density did not change significantly, resulting in a calculated $Z$ number of $\sim 3$ for all the inks. The designations, chemical composition and viscosity of the investigated inks are given in Table 2.

The inks were proved to be chemically stable and produced drops over a period of several months. Their effectiveness for inkjet printing was studied by assessing the properties of the printed structures. After the printed patterns were dried, a

Table 2 Viscosity of the 2MOE-based TAS inks

\begin{tabular}{lll}
\hline Ink & Fraction of viscous solvent vol\% & Viscosity mPa s \\
\hline TAS-GLY & $30 \%$ GLY & 10.1 \\
TAS-PD & $55 \%$ PD & 10.5 \\
TAS-GP05 & $\begin{array}{l}\text { 25\% GLY } \\
\text { T. PD }\end{array}$ & 7.8 \\
& & \\
TAS-GP10 & $25 \%$ GLY & 8.0 \\
& $10 \%$ PD & \\
& & 11.0 \\
TAS-GP15 & $25 \%$ GLY &
\end{tabular}




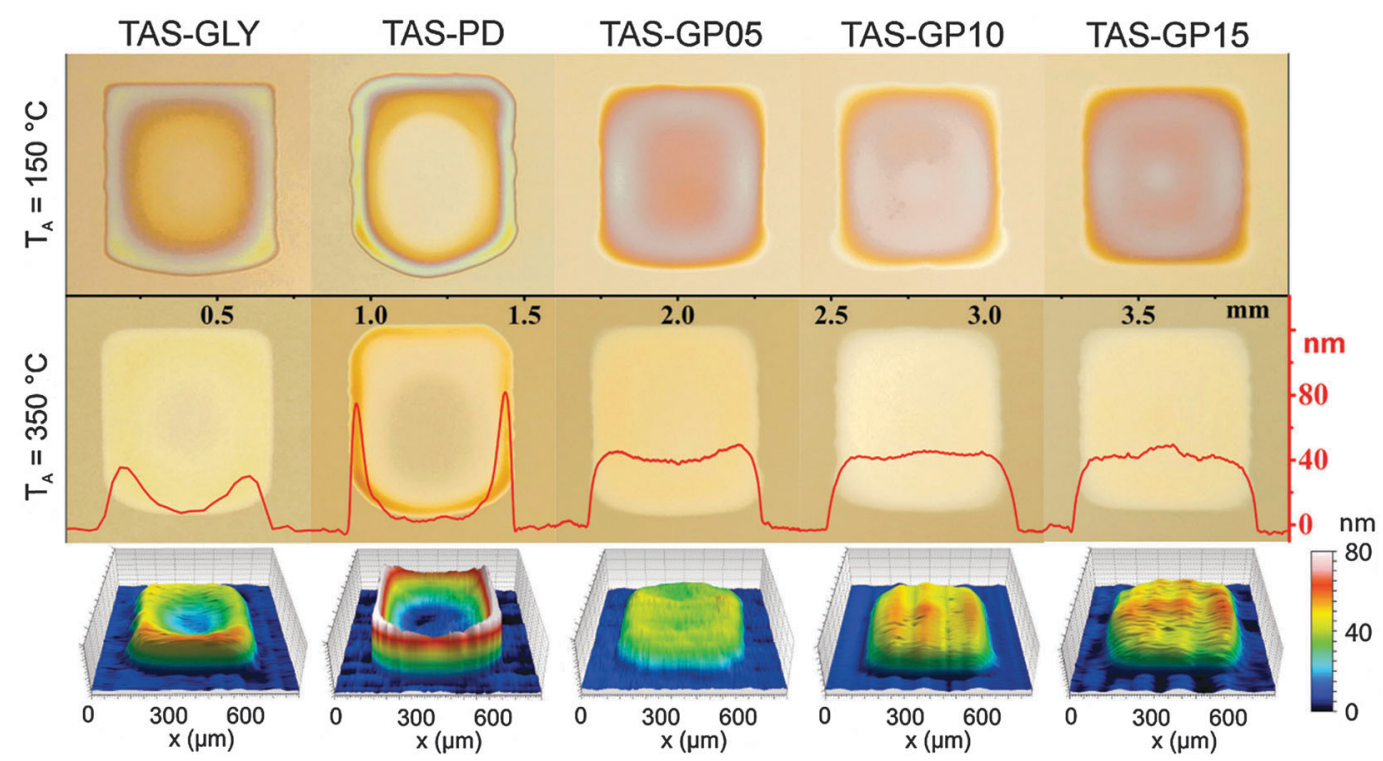

Fig. 2 Optical images of the dried and heated squares and the corresponding profilometry micrographs. The square under investigation was from the central part of an array of $15 \times 15$ squares.

significant difference in the topology could be observed, depending on the ink's solvent composition, as demonstrated in Fig. 2. Drying and heating of both the TAS-GLY and TAS-PD resulted in the coffee-stain effect as shown within the first two columns in Fig. 2.

To overcome the coffee-stain effect, three additional inks were formulated, consisting of all three solvents in such volume ratios that the viscosity, surface tension and $Z$ number were within the "printable range". The viscosity of the inks with $25 \%$ of GLY and 5, 10, or 15\% of PD is between 8 and $11 \mathrm{mPa}$ s, see Table 2, while the surface tension remains at $\sim 34 \mathrm{mN} \mathrm{m}^{-1}$, resulting in a $Z$ number between 3 and 4 . Drying and heating of the ternary-solvent inks resulted in a much flatter surface topography than the structures produced by printing the binary-solvent inks. Moreover, we even observed a shift from a slightly concave to a slightly convex surface topology for the structures printed from the TAS-GP05 and TAS-GP15 inks, respectively, suggesting that the PD could have a strong impact on the drying mechanism. The TAS-GP10 printed structure had a flat surface topology after drying (see the 4th column in Fig. 2).

To understand and explain the phenomena underlying the various topographic features of the printed structures, it is necessary to consider the events that are expected to occur during the drying of the sol-gel ink. In the first possible scenario, the sol-gel transition occurs when the temperature is increased and the viscosity abruptly increases, which "freezes" the shape of the deposit before any significant evaporation occurs (see ref. 26). The second possibility is that the sol-gel transition occurs later on, either just before or after the solvent has completely evaporated, meaning that the ink retains its liquid behaviour throughout the drying process. Our experiments suggest that the sol-gel transition of the investigated inks occurs only at a late stage of the solvent evaporation, which is analogous to the drying of polymer solutions.

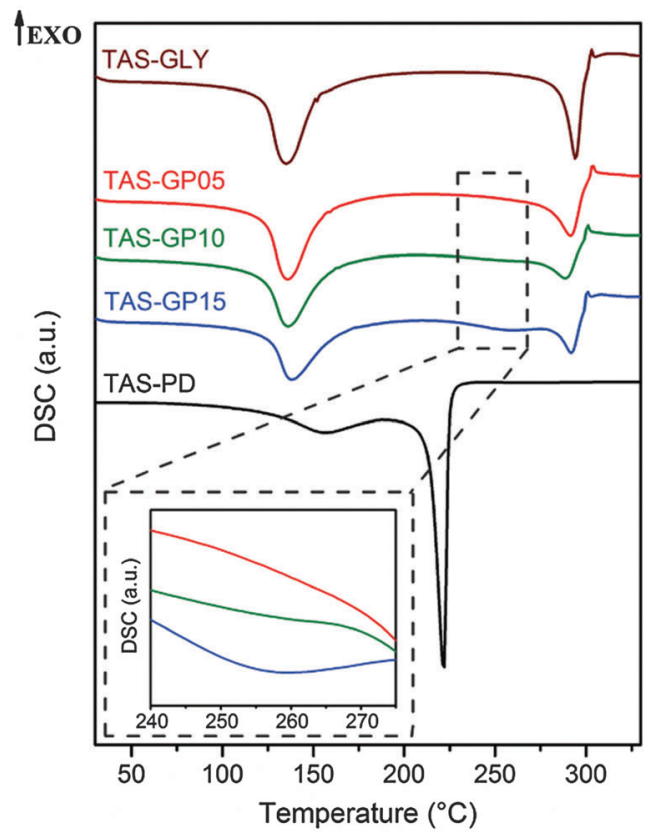

Fig. 3 DSC analysis of the TAS inks with different solvent mixtures. The inset shows the evolution of the endothermic peak in ternary-solvent inks with an increasing amount of PD.

We therefore followed the evaporation process of the TAS inks by recording the enthalpy changes upon heating by DSC (Fig. 3). The endothermic peak at $\sim 135{ }^{\circ} \mathrm{C}$ in the DSC curve of the TASGLY ink is associated with the evaporation of the 2MOE (boiling point: $119{ }^{\circ} \mathrm{C}$ ). For the inks containing both the GLY and PD solvents, the respective peak shifts towards higher temperatures with the increasing fraction of PD. The binary-solvent-based inks exhibited additional peaks at temperatures corresponding to the evaporation of the PD (boiling point: $215{ }^{\circ} \mathrm{C}$ ) or GLY 
(boiling point: $287{ }^{\circ} \mathrm{C}$ ). The glycerol-based inks exhibited an exothermic peak following the endothermic peak at $290{ }^{\circ} \mathrm{C}$, typical for the evaporation associated with the partial decomposition of the GLY. The inset in Fig. 3 reveals a weak and broad endothermic peak appearing at $\sim 260{ }^{\circ} \mathrm{C}$, the intensity of which increased with the increasing fraction of PD and was associated with its evaporation.

Even though several reports on the drying of polymer solutions ${ }^{16,27-31}$ and colloidal suspensions ${ }^{17,32-34}$ emphasize the importance of the flow direction and the velocity on the shape of the dried deposits, the topology of the deposit is not necessarily determined by the flows present in the early stage of drying. In general, there are two flows competing when a binary-solvent ink is drying: the capillary flow towards the periphery of the drying feature and the recirculating Marangoni flow. Two conditions have to be fulfilled for the capillary flow to occur: the contact line pinning and the preferential evaporation of solvents from the edge of the drying feature. ${ }^{32}$ Let us now consider the drying process of a sessile drop composed of two solvents with different boiling points $\left(T_{\mathrm{b}, 1}<T_{\mathrm{b}, 2}\right)$ and surface tensions $\left(\gamma_{1}<\gamma_{2}\right)$. In the initial state, presented in Fig. $4 \mathrm{a}$, the concentrations of the solvents at the edge of the drop ( $x_{1}^{\mathrm{o}}$ and $x_{2}^{\mathrm{o}}$ ) are equal to the ones in the centre of the drop $\left(x_{1}^{i}\right.$ and $\left.x_{2}^{i}\right)$. As the drop dries, the evaporation flux $J$ is higher at the edge of the drop, ${ }^{8}$
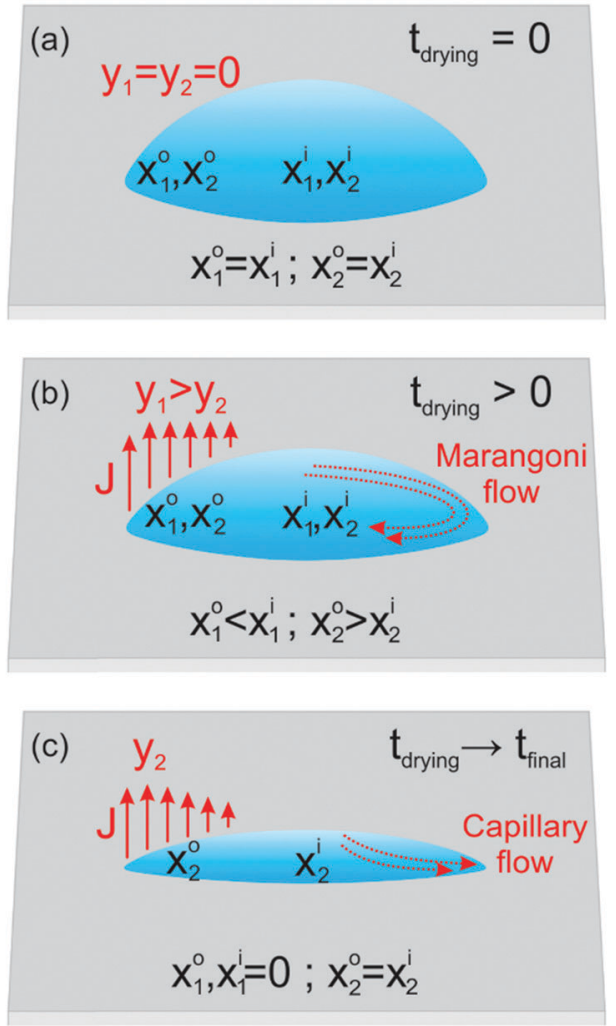

Fig. 4 Schematic presentation of a drop upon drying. The drop consists of two solvents with different volatilities and surface tensions $\left(T_{\mathrm{b}, 1}<T_{\mathrm{b}, 2}\right.$; $\left.\gamma_{1}<\gamma_{2}\right)$ in the (a) initial, (b) intermediate, and (c) late stages. $y_{1}$ and $y_{2}$ are the molar fractions of the solvents 1 and 2 in the vapour phase, respectively. leading to faster evaporation of the more volatile solvent from the edge $\left(y_{1}>y_{2}\right)$, as shown in Fig. $4 \mathrm{~b}$. The concentration gradient is established $\left(x_{1}^{\mathrm{o}}<x_{1}^{i}\right)$ and, due to the different surface tensions of the solvents, an outward Marangoni flow occurs, which often prevails over the capillary flow.

The more volatile solvent might evaporate completely from the drop if the difference in volatility of both solvents is large enough and the fraction of less-volatile solvent is relatively high; this is usually the case for solutions with a low concentration of solute (diluted solutions), where the viscosity has to be increased by adding viscous solvent. In such a situation, the drop would only consist of the less-volatile solvent in the final stage of evaporation and the capillary flow would prevail over the Marangoni flow (Fig. 4c). As a result of the capillary flow in the late stage of drying, a ring-like deposit would be formed.

In our study the binary-solvent inks consist of the $2 \mathrm{MOE}$ with a much lower boiling point (higher volatility) than the GLY or PD. By analogy with Fig. 4, the $2 \mathrm{MOE}$ evaporates faster from the drying features than either of the viscous solvents, resulting in a pronounced coffee-stain effect (see Fig. 2).

Fig. 5 shows the evaporation dynamics of the sessile drops of the TAS-GLY and TAS-PD inks. The drops were observed in the
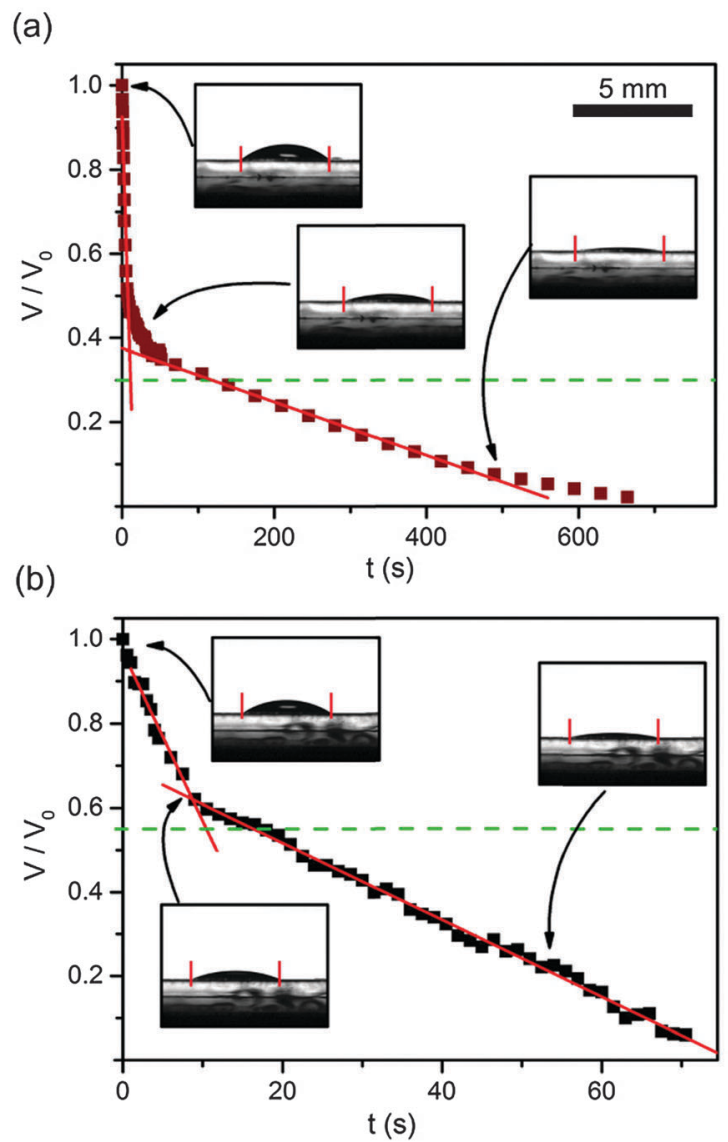

Fig. 5 Normalized volume as a function of time during the drying process of (a) TAS-GLY and (b) TAS-PD drops. The green dotted line corresponds to the initial volume fraction of the less-volatile solvent. Note the different timescales in (a) and (b). Photographs of sessile drops at selected times are included. 
cross-section view while drying at $150{ }^{\circ} \mathrm{C}$. Note that the drops appear to be self-pinned, as shown in the insets of Fig. 5. Two distinct stages of evaporation can be observed. In the first stage $(t \approx 30 \mathrm{~s}$ for TAS-GLY and $t \approx 10 \mathrm{~s}$ for TAS-PD) the drop volume decreased rapidly, and the volume change was proportional to the fraction of $2 \mathrm{MOE}$ in both inks. In the second stage, the lessvolatile solvent evaporated. Due to the lower boiling point and thus a higher volatility, the PD-based ink dried significantly faster $\left(t_{\text {drying }}=68 \mathrm{~s}\right)$ than the glycerol-based ink $\left(t_{\text {drying }}=700 \mathrm{~s}\right)$. The fact that $2 \mathrm{MOE}$ evaporates faster from the drying features can moreover explain why the TAS-PD-printed patterns show a much more pronounced edge deposit than the TAS-GLY as demonstrated in Fig. 2. As the drying proceeds and the fraction of the viscous solvent increases, the latter starts to dominate the drying process; the drying of TAS-PD and TAS-GLY inks becomes governed by the properties of PD and GLY, respectively. It has been shown that the coffee-stain effect is reduced for highly viscous systems (as in the case of GLY). ${ }^{35}$

It is evident from Fig. 2 that by introducing the third viscous solvent, i.e. adding the PD to the TAS-GLY ink, the formation of a coffee stain is inhibited and flat structures can be printed by carefully adjusting the solvent composition of the ink.

\section{Printed capacitors}

As a proof of concept, we fabricated thin-film capacitors by inkjet printing the TAS-GP10 ink on ITO-coated glass and sputtering top Au electrodes. The optical photograph of the structure is shown in Fig. 6a.

The TAS dielectric layer exhibited a smooth, flat surface (Fig. 6b), without any pinholes, cracks, or grain boundaries, as confirmed by AFM (Fig. 6c). The root mean square (RMS) roughness of the surface was $210 \mathrm{pm}$, which is about two times less than the surface roughness of the ITO substrate $(470 \mathrm{pm})$. The AFM analysis also tentatively suggests that the TAS structures are
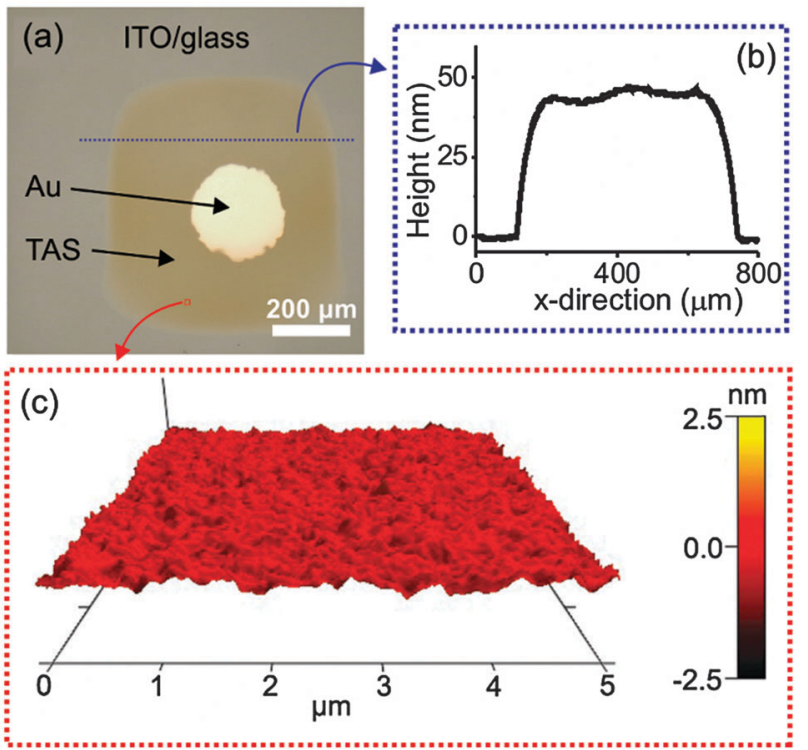

Fig. 6 (a) Optical photograph, (b) cross-sectional profile and (c) 3D AFM topology image of $45 \mathrm{~nm}$-thick printed TAS-GP10 thin-film capacitors. amorphous, an observation supported by previous reports for films that were deposited by spin coating and heated at the same temperature of $350{ }^{\circ} \mathrm{C} .^{20}$

The dielectric constant $\left(\varepsilon_{\mathrm{r}}\right)$ of the printed capacitors was $\sim 15$ and $\tan (\delta) \sim 0.033$ at $100 \mathrm{kHz}$. We noticed a weak frequency dispersion in the frequency range of $10^{2}-10^{6} \mathrm{~Hz}$ (Fig. 7a). The increase in the dielectric losses, and concomitantly the dielectric constant, at low frequencies could be attributed to the space-charge polarization, as previously observed for solution-processed $\mathrm{Ta}_{2} \mathrm{O}_{5}$ films. ${ }^{36}$ At high frequencies the dielectric losses increase and the dielectric constant starts to decrease. This is most probably an extrinsic effect caused by the high resistance of the ITO-covered glass $\left(R_{\mathrm{S}} \sim 200 \Omega \square^{-1}\right)$, which functions as the bottom electrode of the metal-insulator-metal structured capacitors. It is known that a resistor connected in series with a leaky capacitor introduces a Debye-like response, i.e. a decrease of the dielectric constant and a peak in the dielectric losses. $^{37}$

The leakage characteristics are presented in Fig. 7b. The leakage-current density increases non-linearly with the electric field and exhibits a value of $2.4 \times 10^{-7} \mathrm{~A} \mathrm{~cm}^{-2}$ at an electric field of $200 \mathrm{kV} \mathrm{cm}{ }^{-1}$. The commonly observed conduction mechanisms in $\mathrm{Ta}_{2} \mathrm{O}_{5}$ thin films are Schottky emission at low electric fields and Poole-Frenkel emission at high fields. ${ }^{38,39}$

(a)

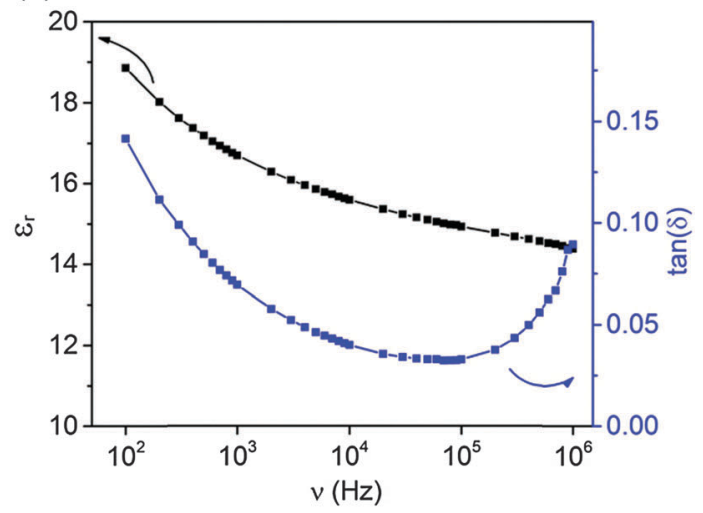

(b)

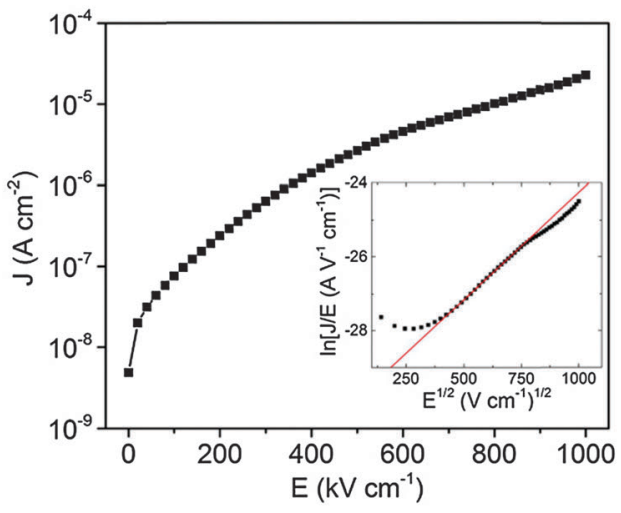

Fig. 7 (a) Dispersion of the dielectric constant and losses with frequency and (b) leakage-current density as a function of the applied electric field for a $45 \mathrm{~nm}$-thick printed TAS capacitor. The inset in (b) shows the PooleFrenkel plot $\left(\ln (J / E)-E^{1 / 2}\right)$. 
The linear fit in the $\ln (J / E)-E^{1 / 2}$ plot shown in the inset of Fig. $7 \mathrm{~b}$ is in agreement with the Poole-Frenkel conduction mechanism.

The performance of our printed capacitors is comparable with about-three-times-thicker $(135 \mathrm{~nm})$ spin-coated films of the same composition heated at $350{ }^{\circ} \mathrm{C},{ }^{40}$ demonstrating the viability of the ink design discussed in this article.

\section{Conclusions}

Additive deposition techniques such as inkjet printing represent one of the key routes to micro/nanoscale design and lower production costs for electronic devices; however, the deficiency of the control over the uniformity of the dried deposits represents a major impediment to the production of reliable devices. This work presents a detailed study of the critical dependence of the deposit geometry on the ink formulation. The fundamental significance of the ink parameters is highlighted and examined through an evaluation of the links between the solvents used, the drying mechanisms and the drying rates.

By modifying the solvent composition of the inks, we were able to tailor the topology of the inkjet-printed structures. The optimized inks resulted in high-quality dielectric structures with a well-defined geometry and functional properties comparable to spin-coated films.

\section{Acknowledgements}

We acknowledge Dr Alja Kupec for measurements of the physical properties of the solvent mixtures and the financial support of the Slovenian Research Agency (J2-4173, P2-0105, PR-06799).

\section{Notes and references}

1 R. N. Das, H. T. Lin, J. M. Lauffer and V. R. Markovich, Circuit World, 2011, 37, 38-45.

2 A. Kamyshny and S. Magdassi, Small, 2014, 10, 3515-3535.

3 M. Singh, H. M. Haverinen, P. Dhagat and G. E. Jabbour, Adv. Mater., 2010, 22, 673-685.

4 T. H. J. van Osch, J. Perelaer, A. W. M. de Laat and U. S. Schubert, Adv. Mater., 2008, 20, 343-345.

5 B. J. de Gans, P. C. Duinevelt and U. S. Schubert, Adv. Mater., 2004, 16, 203-213.

6 D. Jang, D. Kim and J. Moon, Langmuir, 2009, 25, 2629-2635.

7 N. Reis and B. Derby, Mater. Res. Soc. Symp. Proc., 2000, 625, 117-122.

8 R. D. Deegan, O. Bakajin and T. F. Dupont, Nature, 1997, 389, 827-829.

9 K. Fukuda, T. Sekine, D. Kumaki and S. Tokito, ACS Appl. Mater. Interfaces, 2013, 5, 3916-3920.

10 J. Sun, B. Bao, M. He, H. Zhou and Y. Song, ACS Appl. Mater. Interfaces, 2015, 7, 28086-28099.

11 M. Kuang, L. Wang and Y. Song, Adv. Mater., 2014, 26, 6950-6958.
12 C. Kim, M. Nogi and K. Suganuma, J. Micromech. Microeng., 2012, 22, 35016-35020.

13 B. J. Kang and J. H. Oh, Thin Solid Films, 2010, 518, 2890-2896.

14 M. Majumder, C. S. Rendall, J. A. Eukel, J. Y. L. Wang, N. Behabtu, C. L. Pint, T.-Y. Liu, A. W. Orbaek, F. Mirri, J. Nam, A. R. Barron, R. H. Hauge, H. K. Schmidt and M. Pasquali, J. Phys. Chem. B, 2012, 116, 6536-6542.

15 P. J. Yunker, T. Still, M. A. Lohr and A. G. Yodh, Nature, 2011, 476, 308-311.

16 E. Tekin, B. J. Gans and U. S. Schubert, J. Mater. Chem., 2004, 14, 2627-2632.

17 J. Park and J. Moon, Langmuir, 2006, 22, 3506-3513.

18 J. Sun, B. Bao, J. Jiang, M. He, X. Zhang and Y. Song, $R S C$ Adv., 2016, 6, 31470-31475.

19 C. Chaneliere, J. L. Autran, R. A. B. Devine and B. Balland, Mater. Sci. Eng., R, 1998, 22, 269-322.

20 R. C. Frunză, B. Kmet, M. Jankovec, M. Topič and B. Malič, Mater. Res. Bull., 2014, 50, 323-328.

21 P. Barquinha, L. Pereira, G. Gonçalves, R. Martins, E. Fortunato, D. Kuščer, M. Kosec, A. Vilà, A. Olziersky and J. R. Morante, J. Soc. Inf. Disp., 2010, 18, 762-772.

22 J. Tellier, B. Malič, D. Kuščer, G. Trefalt and M. Kosec, J. Am. Ceram. Soc., 2011, 94, 2834-2840.

23 D. Kuščer, G. Stavber, G. Trefalt and M. Kosec, J. Am. Ceram. Soc., 2012, 95, 487-493.

24 Fujifilm Dimatix. Jettable Fluid Formulation Guidelines, https://www.fujifilmusa.com/shared/bin/Dimatix-MaterialsPrinter-Jettable-Fluid-Formulation-Guidelines.pdf (accessed February 2016).

25 L. Grunberg and A. H. Nissan, Nature, 1949, 164, 799-800.

26 A. M. J. Berg, A. W. M. Laat, P. J. Smith, J. Perelaer and U. S. Schubert, J. Mater. Chem., 2007, 17, 677-683.

27 E. Tekin, P. J. Smith and U. S. Schubert, Soft Matter, 2008, 4, 703-713.

28 T. Kajiya and M. Doi, Nihon Reoroji Gakkaishi, 2011, 39, 17-28.

29 P. O. Babatunde, W. J. Hong, K. Nakaso and J. Fukai, AIChE J., 2013, 59, 699-702.

30 J. Fukai, H. Ishizuka, Y. Sakai, M. Kaneda, M. Morita and A. Takahara, Int. J. Heat Mass Transfer, 2006, 49, 3561-3567.

31 C. Poulard and P. Damman, Europhys. Lett., 2007, 80, 1-5.

32 R. Deegan, O. Bakajin, T. Dupont, G. Huber, S. R. Nagel and T. Witten, Phys. Rev. E: Stat. Phys., Plasmas, Fluids, Relat. Interdiscip. Top., 2000, 62, 756-765.

33 G. Berteloot, A. Hoang, A. Daerr, H. P. Kavehpour, F. Lequeux and L. Limat, J. Colloid Interface Sci., 2012, 370, 155-161.

34 R. Dou, T. Wang, Y. Guo and B. Derby, J. Am. Ceram. Soc., 2011, 94, 3787-3792.

35 A. Friederich, J. R. Binder and W. Bauer, J. Am. Ceram. Soc., 2013, 96, 2093-2099. 
36 S. Yildirim, K. Ulutas, D. Deger, E. O. Zayim and I. Turhan, Vacuum, 2005, 77, 329-335.

37 A. Eršte, B. Malič, B. Kužnik, M. Kosec and V. Bobnar, Equivalent Circuit in Modeling of Core-Shell Structured Ceramic Materials, Electroceramics II: Ceramic Transactions, ed. K. M. Nair and S. Priya, Wiley, Hoboken, NJ, 2012, pp. 23-29.
38 E. Atanassova and A. Paskaleva, Microelectron. Reliab., 2002, 42, 157-173.

39 F. C. Chiu, J. J. Wang, J. Y. M. Lee and S. C. Wu, J. Appl. Phys., 1997, 81, 6911-6915.

40 R. C. Frunză, M. Jankovec, B. Kmet, M. Topič and B. Malič, Journal of Microelectronics, Electronic Components and Materials, 2014, 44, 19-24. 